\title{
The phosphoinositide 5-phosphatase INPP5K: from gene structure to in vivo functions
}

Stéphane Schurmans ${ }^{a, b^{*}}$, Charles-Andrew Vande Catsyne ${ }^{a}$, Christophe Desmet ${ }^{c}$, Bastien Moësa

aLaboratoire de Génétique Fonctionnelle, GIGA-Research Centre, building B34, CHU Sart-Tilman, Université de Liège, avenue de l'Hôpital 11, 4000-Liège, Belgium bSecteur de Biochimie Métabolique vétérinaire, Département des Sciences Fonctionnelles, Faculté de Médecine Vétérinaire, building B42, Université de Liège, Quartier Vallée 2, avenue de Cureghem 7A-7D, 4000-Liège, Belgium 'Laboratory of Cellular and Molecular Immunology, GIGA-Research Centre, building B34, CHU Sart-Tilman, Université de Liège, avenue de l'Hôpital 11, 4000-Liège, Belgium.

\section{${ }^{*}$ Corresponding author :}

Laboratoire de Génétique Fonctionnelle, GIGA-Research Centre, building B34, CHU Sart-Tilman, Université de Liège, avenue de l'Hôpital 11, 4000-Liège, Belgium; phone : +32 43663372 ; email : sschurmans@uliege.be

Keywords: inositide, phosphoinositide, 5-phosphatase, INPP5K, SKIP, phosphatidylinositol 3,4,5-trisphosphate, phosphatidylinositol 4,5-bisphosphate, congenital muscular dystrophy, cataract, intellectual disability, insulin signaling, insulin resistance, endoplasmic reticulum, endoplasmic reticulum stress, unfolded protein response 
Abbreviations: ER: endoplasmic reticulum; Ptdlns(3,4,5)P3 : phosphatidylinositol 3,4,5-trisphosphate ; Ptdlns(4,5)P2 : phosphatidylinositol 4,5-bisphosphate; UPR: unfolded protein response 


\section{Abstract :}

INPP5K (Inositol Polyphosphate 5-Phosphatase K, or SKIP (for Skeletal muscle and Kidney enriched Inositol Phosphatase) is a member of the phosphoinositide 5phosphatases family. Its protein structure is comprised of a $\mathrm{N}$-terminal catalytic domain which hydrolyses both Ptdlns(4,5)P2 and Ptdlns(3,4,5)P3, followed by a $\mathrm{SKICH}$ domain at the $\mathrm{C}$-terminus which is responsible for protein-protein interactions and subcellular localization of INPP5K. Strikingly, INPP5K is mostly concentrated in the endoplasmic reticulum, although it is also detected at the plasma membrane, in the cytosol and the nucleus. Recently, mutations in INPP5K have been detected in patients with a rare form of autosomal recessive congenital muscular dystrophy with cataract, short stature and intellectual disability. INPP5K functions extend from control of insulin signaling, endoplasmic reticulum stress response and structural integrity, myoblast differentiation, cytoskeleton organization, cell adhesion and migration, renal osmoregulation, to cancer. The goal of this review is thus to summarize and comment recent and less recent data in the literature on INPP5K, in particular on the structure, expression, intracellular localization, interactions and functions of this specific member of the 5-phosphatases family. 


\section{Introduction:}

Phosphatidylinositol (Ptdlns) is composed of a glycerol backbone esterified to two fatty acid chains and a phosphate, and attached to a polar head group, the cyclic polyol myo-inositol, that extends into the cytoplasm. This inositol head group has free hydroxyl groups at positions D2 through D6, and those at positions D3, D4 and D5 can be phosphorylated into 7 potential combinations by 3-, 4- and 5-kinases, respectively. The 7 phosphorylated forms of phosphatidylinositol are named phosphoinositides, are present in cell membranes and play important role in many biological processes, essentially by interacting with specific proteins (Grabon et al., 2019). Indeed, each cellular membrane compartment has a characteristic composition of phosphoinositide species, and this phosphoinositide signature attracts a specific set of functionally important proteins to that membrane, or directly regulates the activity level of integral membrane proteins (Falkenburger et al., 2010). Beside kinases, phosphoinositide metabolism is also regulated by members of phospholipases $\mathrm{C}$ and phosphatases families. Phosphates added at positions D3, D4 and D5 of the inositol head group by kinases can be removed by 3-, 4- and 5phosphatases, respectively (Blero et al., 2007). The mammalian 5-phosphatases family comprises 10 members. These members share an inositol 5-phosphatase domain with a set of conserved amino acids and a general comparable structural organization. Except for one member, INPP5A, they all catalyze the dephosphorylation of Ptdlns(4,5)P2 and/or Ptdlns(3,4,5)P3 at the 5- position, generating Ptdlns(4)P and/or Ptdlns(3,4)P2, respectively (Ramos et al., 2019a). Interestingly, 5-phosphatase isoenzymes have been individually implicated in human diseases, such as ciliopathies (INPP5E), the oculo-cerebrorenal syndrome of Lowe (INPP5F) or opsismodysplasia, a rare congenital skeletal dysplasia (INPPL1) (Ramos 
et al., 2019). The goal of this review is to summarize and comment recent and less recent data in the literature on INPP5K, in particular on the in vivo function of this member of the 5-phosphatases family in man and genetically-modified animals. 


\section{Official full name/symbol and synonym:}

The official full name for the INPP5K gene is Inositol Polyphosphate 5-Phosphatase K. However, SKIP (for Skeletal muscle and Kidney enriched Inositol Phosphatase) was first used when this 5-phosphatase was discovered by Takenawa's team in 2000 (ljuin et al., 2000).

\section{INPP5K gene, transcripts and proteins in man and mouse:}

The human INPP5K gene is located on the telomeric region of the short arm of chromosome 17 (17p13.3), on the reverse strand between nucleotides $1,494,577$ and 1,516,742. The gene comprises 12 exons and generates a 2,706 bp transcript coding for a 448 amino acids protein with a theoretical $51.1 \mathrm{kDa}$ molecular mass (Figure 1; e!Ensembl). Many splice variants of INPP5K are reported in public databases, giving potential proteins of 84 to 229 amino acids in length. However, the in vivo relevance of these transcripts is currently unknown. By contrast, two interesting transcripts containing insertions of 231 and 390 bp between exon 1 and 2 of the 2,706 bp transcript described above have been reported (ljuin et al., 2000; e!Ensembl). They both lead to the production of the same 372 amino acids $/ 42.7 \mathrm{kDa}$ protein that lacks the first 76 amino acids of the $51.1 \mathrm{kDa}$ INPP5K protein, probably by using a second Start codon present in exon 3. Both $\sim 51.1$ and $\sim 42.7 \mathrm{kDa}$ signals were detected in tissue and cell line protein extracts by Western blotting using an affinity-purified rabbit antibody directed against amino acid residues 137-448 of the human INPP5K protein (ljuin et al., 2000). An additional $~ 36 \mathrm{kDa}$ signal of unknown amino-acid sequence was also detected by Western blotting using an affinity-purified rabbit antibody raised against a fusion peptide representing the $\mathrm{N}$ - and $\mathrm{C}$-terminal seven amino acids of human 51.1 kDa INPP5K protein (Gurung et al., 2003). 
In mouse, the Inpp5k gene is located on the long arm of chromosome 11 (11qB5), on the forward strand between nucleotides $75,630,988$ and $75,648,871$. The mouse Inpp5k gene also comprises 12 exons, as in man, and generates a 2,679 bp transcript coding for a 468 amino acids protein with a theoretical molecular mass of $54.2 \mathrm{kDa}$ (e!Ensembl). Two splice variants giving potential proteins of 39 and 112 amino acids in length are reported in the databases, but their in vivo relevance is unknown. When mouse tissues protein extracts were probed by Western blotting with an affinity-purified rabbit antibody raised against the RSFLREDTLYEPEPQI carboxyterminal peptide of the INPP5K mouse protein, $\sim 54$ and $\sim 42 \mathrm{kDa}$ signals were clearly detected (Pernot et al., 2011). A third $\sim 51 \mathrm{kDa}$ signal was specifically observed in testis and brain and, in the kidney, a fourth $\sim 36 \mathrm{kDa}$ signal was also present in addition to the $\sim 42 \mathrm{kDa}$ signal (Pernot et al., 2011). The exact amino-acid sequence and origin of these 51, 42 and $36 \mathrm{kDa}$ signals are currently unknown.

In conclusion, these results indicate that mouse and human INPP5K genes have a very similar structure and lead to the production of full length 54.2 and $51.1 \mathrm{kDa}$ catalytically active INPP5K proteins, respectively. Additional truncated INPP5K proteins probably also arise from either alternative splicing, the use of alternative Start codon or simply from partial proteolysis, which are also detected by Western blotting with specific antibodies. The exact biological activity of these truncated proteins is currently unknown.

\section{Expression of INPP5K mRNAs and proteins in human and mouse tissues and} cells:

Results from Northern blotting, RT-PCR and Western blotting indicate that expression of INPP5K mRNA and protein is rather ubiquitous in human and mouse tissues and cells lines (ljuin et al., 2000; Pernot et al., 2011). Importantly, INPP5K is 
particularly abundant in skeletal muscle, heart, brain, kidney, testis and eye (see below). Hematopoietic cells also express INPP5K mRNA and protein, including resting and activated lymphocytes, neutrophils, macrophages and dendritic cells (Pernot et al., 2011).

In addition to the full length $54.2 \mathrm{kDa}$ mouse and $51.1 \mathrm{kDa}$ human INPP5K proteins, mouse 51,42 and $36 \mathrm{kDa}$ signals as well as human 42.7 and $36 \mathrm{kDa}$ signals were detected by Western blotting (ljuin et al., 2000; Gurung et al., 2003; Pernot et al., 2011). Intriguingly, tissues and cell lines express either a single INPP5K protein or a combination of full length and/or truncated INPP5K proteins. For example, the mouse $\mathrm{C} 2 \mathrm{C} 12$ cell line and mouse ovary only express the $42 \mathrm{kDa}$ truncated protein. By contrast, full length $54.2 \mathrm{kDa}$ and truncated $42 \mathrm{kDa}$ proteins are both detected in mouse spleen. The most intriguing combination is probably in the mouse kidney where a very strong $36 \mathrm{kDa}$ signal is associated with faint 54.2, 51 and 42 signals in the cortex, whereas faint 42 and $36 \mathrm{kDa}$ signals are detected in the medulla (Pernot et al., 2011).

Physiological and physiopathological conditions associated with an increased INPP5K mRNA/protein expression include skeletal muscle cell differentiation (Xiong et al., 2011; ljuin and Takenawa, 2012a), endoplasmic reticulum stress and activation of the unfold protein response (ljuin et al., 2015a) as well as insulin resistance, obesity and diabetes (ljuin et al., 2015a). In cancer, INPP5K mRNA expression was found either significantly increased (endometrial carcinoma (Hedberg Oldfors et al., 2015), prostate cancer (Flaig et al., 2017), glioblastoma (Davies et al., 2015), renal cancer (Jones et al., 2005)) or decreased (lung adenocarcinoma (Gao et al., 2017), glioblastoma (Davies et al., 2015)), depending on the cancer cell type.

Human and mouse INPP5K protein structure and catalytic activity: 
When compared to the human 448 amino-acids $/ 51.1 \mathrm{kDa}$ INPP5K protein, the mouse 468 amino-acids/54.2 $\mathrm{kDa}$ INPP5K protein is characterized by the presence of an additional peptide of 18 residues of unknown function at the $\mathrm{N}$-terminus. With the exception of this peptide at the $\mathrm{N}$-terminus, human and mouse INPP5K protein structure is identical and simple: a catalytic domain comprising of the two conserved and specific motifs of the 5-phosphatases family is present at the amino-terminus of the protein (residues 12-326 of the human $51.1 \mathrm{kDa}$ protein and 30-345 of the murine $54.2 \mathrm{kDa}$ protein), followed by a SKICH domain at the carboxy-terminus (residues 332-431 of the human $51.1 \mathrm{kDa}$ protein and $351-450$ of the murine $54.2 \mathrm{kDa}$ protein) (Figure 1). Accordingly, the human 372 amino acids/42.7 kDa protein described above, which lacks the first 76 amino acids of the $51.1 \mathrm{kDa}$ full length INPP5K protein, is unlikely to harbor 5-phosphatase activity. INPP5K hydrolyses both Ptdlns(4,5)P2 and Ptdlns(3,4,5)P3, but exhibits greatest activity towards Ptdlns(4,5)P2 (ljuin et al., 2000; Gurung et al., 2003; Schmid et al., 2004; Pernot et al., 2011). No phosphatase activity was detected against Ptdlns(3,5)P2, Ptdlns(5)P, Ins(1,4,5)P3 or Ins(1,3,4,5)P4 (Gurung et al., 2003; Schmid et al., 2004).

The 100 amino-acid SKICH domain (for SKIP carboxy-homology domain) has an Iglike $\beta$-barrel structure and a molecular surface dominated by negatively charged regions which may interact with components of the plasma membrane (Yang et al., 2015). The SKICH domain and surrounding sequences present at the C-terminus of INPP5K mediates interactions with specific proteins and regulate intracellular localization (Gurung et al., 2003; Dong et al., 2018). For example, after growth factor/insulin stimulation, the SKICH domain mediates INPP5K translocation from its endoplasmic reticulum localization in basal conditions to the plasma membrane. The 
SKICH domain of INPP5K is composed of a core sequence allowing a constitutive plasma membrane association and of surrounding sequences specific to INPP5K that contribute to endoplasmic reticulum localization of the protein in basal conditions (Gurung et al., 2003, Dong et al., 2018).

\section{INPP5K interacting proteins:}

Lists of proteins potentially interacting with the INPP5K protein are available in BioGRID (37 potential interactors), CORUM (1 potential complex: ID 2639, the HES1 promoter-Notch enhancer complex), IntAct (38 potential interactors), MINT (7 potential interactors) and STRING (10 predicted functional partners) public databases and are summarized on www.uniprot.org/uniprot/Q9BT40. The present review will be limited to 6 interacting proteins which have been carefully analyzed by different teams as part of specific studies on INPP5K.

GRP78/BiP/HSP5a and activated PAK1:

ljuin and collaborators proposed a model in which, in basal conditions, cytosolic INPP5K directly interacts via its SKICH domain with GRP78/Glucose regulated protein 78, a luminal ER chaperone protein involved in ER stress and the UPR (ljuin and Takenawa, 2012b, 2015a, 2015b, 2015c and 2016). However, in this model, the unresolved issue is how INPP5K which is present in the cytosol and at the cytosolic side of the ER membrane interacts with luminal ER GRP78 (ljuin et al., 2016). After insulin stimulation, the INPP5K-GRP78 complex migrates from ER to the plasma membrane, where activated PAK1/p21-activated protein kinase 1 competitively binds through a 11 amino-acids peptide within its kinase domain to INPP5K in place of GRP78, linking INPP5K to the complex of proteins associated with the insulin receptor. Binding of INPP5K to activated PAK1 and this complex via its $\mathrm{SKICH}$ domain leads to Ptdlns( $3,4,5)$ P3 dephosphorylation, decreased AKT2 
phosphorylation and insulin signaling inactivation (see below for the physiologic effect of this regulation). The co-immunoprecipitation of expressed tagged INPP5K and GRP78 proteins was reproduced by another team (Wiessner et al., 2017). SODD/BAG4:

According to Rahman and collaborators, the direct interaction between the molecular co-chaperone SODD/Silencer of Death Domains and INPP5K following growth factor stimulation exerts an inhibitory effect on INPP5K Ptdlns(3,4,5)P3 5-phosphatase catalytic activity. Consequently, this interaction enhances the recruitment of Ptdlns(3,4,5)P3-effectors at the plasma membrane and reduces that of Ptdlns(3,4)P2, resulting in a tight regulation of actin polymerization, cell adhesion, spreading and migration (Rahman et al., 2011). Upon growth factor stimulation, SODD co-localized with INPP5K both in the cytosol and at the plasma membrane. No interaction was detected in basal conditions. In contrast, $S O D D^{-/}$mouse embryonic fibroblasts exhibited reduced AKT phosphorylation following growth factor stimulation, associated with increased INPP5K Ptdlns(3,4,5)P3-5-phosphatase activity (Rahman et al., 2011).

\section{ARL6IP1:}

ARL6IP1/ADP Ribosylation Factor Like GTPase 6 Interacting Protein 1 is a protein with reticulon-like features whose mutations result in hereditary spastic paraplegia, a pathology frequently associated with dysfunction of proteins that control ER morphology (Blackstone et al., 2011; Novarino et al., 2014; Yamamoto et al, 2014; Nizon et al., 2018). ARL6IP1 is embedded in the ER membrane via 4 transmembrane domains but its $\mathrm{N}$ - and C-terminal regions protrude in the cytosol (Dong et al., 2018). Dong and collaborators convincingly demonstrated that the interaction with ER ARL6IP1 protein is responsible for INPP5K recruitment from the cytosol to the 
cytosolic side of the ER membrane and that, like ARL6IP1, INPP5K is enriched in newly formed ER tubules. Importantly, knockdown of either protein resulted in structural ER alterations (Dong and al., 2018; Ramos et al., 2020). The ARL6IP1 cytosolic N-terminal region and a surface of INPP5K at the interface of the catalytic domain and the SKICH domain were implicated in the INPP5K-ARL6IP1 interaction (Dong et al., 2018).

\section{MAD2L1BP/p31(comet)/CMT2:}

Yeast-two-hybrid analysis revealed MAD2L1BP/MAD2L1 binding protein, a nuclearenriched protein binding to the mitotic spindle assembly checkpoint MAD2A protein during cell cycle surveillance, as a potential INPP5K interactor (Rual et al., 2005). In EGFP-INPP5K-expressing HeLa cells, mCherry-MAD2L1BP co-expression resulted in a massive translocation of INPP5K from ER to the nucleus and in a marked nuclear INPP5K accretion (Dong et al., 2018). However, the interaction between native proteins was not analyzed and interacting domains in INPP5K and MAD2L1BP proteins were not defined. Thus, the physiological relevance of this interaction remains to be defined.

2/5C HBV core protein:

Interestingly, the SKICH domain of INPP5K was reported to interact with amino acids 116-149 of the 2/5C human hepatitis B virus (HBV) core protein (Hung et al., 2009). The interaction with 2/5C HBV core protein induces INPP5K migration from ER to the nucleus.

\section{Intracellular localization of INPP5K:}

In non-stimulated conditions, immunofluorescence studies revealed that both endogenous and exogenous tagged INPP5K proteins are mostly concentrated in the ER, co-localizing with calreticulin and calnexin ER markers, and that little or no 
staining is detected at the plasma membrane (ljuin et al., 2000; Gurung et al., 2003, Dong et al., 2018, Ramos et al., 2018, 2020). As mentioned above, at the cytosolic side of the ER membrane, INPP5K binds to the N-terminal region of ARL6IP1 which protrudes in the cytosol (Dong et al., 2018). Interaction of INPP5K with luminal ER GRP78 has also been described, but the molecular mechanism is still unresolved (ljuin et al., 2016). It is noteworthy here that mammalian INPP5K shares this ER localization with the yeast phosphoinositide 5-phosphatase Inp54p. Inp54p is a 44 kDa protein which consists of a N-terminal Ptdlns(4,5)P2 5-phosphatase domain and a C-terminal leucine-rich tail that is sufficient to target the protein to the cytosolic side of the ER membrane (Wiradjaja et al., 2001). After growth factor or insulin stimulation, INPP5K is transiently translocated from the ER to the plasma membrane, where it could bind to activated PAK1, SODD or still undefined plasma membrane ligands via its SKICH domain (see above). At the plasma membrane, INPP5K colocalizes with submembranous actin at the leading edge of the cell, specifically at membrane ruffles (Gurung et al., 2003; ljuin and Takenawa, 2003, 2012b, 2016). Finally, Ramos and collaborators recently reported that INPP5K, beside its known ER, plasma membrane and cytosolic localizations, is also present in the nucleus of the human U-251 malignant glioblastoma (MG) cell line (Ramos et al., 2020). In this cell line, about half of the nuclear INPP5K staining co-localized with SC- $35^{+}$speckles, where Ptdlns(4,5)P2 has been previously detected.

INPP5K mutations are responsible for a rare form of autosomal recessive congenital muscular dystrophy with cataract, short stature and intellectual disability in man:

In 2017, 2 research teams reported that biallelic mutations in the INPP5K gene cause a form of congenital muscular dystrophy with early-onset cataract, short stature and 
cognitive impairment (gene/locus MIM: 607875; phenotype MIM: 617404) (Wiessner et al., 2017; Osborn et al., 2017). A total of 9 causative INPP5K alleles were identified: 7 in the catalytic region and 2 in the SKICH domain (Figure 1). All 7 catalytic mutants and one of the SKICH domain mutant (p.Asn417Lysfs²6) exhibited moderate to severe decrease in Ptdlns(4,5)P2 phosphatase activity in vitro, as compared with control INPP5K protein (Wiessner et al., 2017; Osborn et al., 2017). The second SKICH domain mutant, p.lle363Thr, had normal PtdIns(4,5)P2 phosphatase activity but displayed a striking tendency for a more diffuse intracellular distribution in transfected cells than control INPP5K protein (Wiessner et al., 2017). It is noteworthy here that patients homozygous for the p.lle50Thr catalytic mutant had a variable penetrance for muscular dystrophy and intellectual disability, leaving in one patient the ocular manifestation as the main phenotype and suggesting a role for epigenetic factors and/or genetic modifiers in the phenotype (Yousaf et al., 2018). Electromyography showed myopathic changes in the muscles of all affected individuals tested, in association with normal sensory and motor nerve conduction. Muscle pathology was largely non-specific, showing variable degrees of dystrophic features, including increased range of muscle fiber size and diameter, muscle fibrosis, excess adipose tissue, occasional fibers with centrally located nuclei or vacuoles, rare necrotic fibers, and a few basophilic regenerating fibers. There were no abnormalities of blood vessels, inflammatory changes, or group atrophy. Immunohistochemical analysis of classical skeletal muscle markers was normal, except for $\alpha$-dystroglycan expression, which was reduced in 2 of the 12 affected individuals tested (Wiessner et al., 2017; Osborn et al., 2017). Transmission electron microscopy revealed several fibers with pronounced reduction of myofibrils and disrupted Z line material (Wiessner et al., 2017). Unfortunately, attempts to identify 
alterations in biological processes in which INPP5K had been previously implicated (see below) were unsuccessful, and thus the pathogenic mechanism of this rare congenital muscle disease remains totally unknown. Indeed, skin fibroblasts from individuals homozygous for the p.lle50Thr INPP5K mutation and from control subjects showed no difference in AKT phosphorylation in response to IGF-II stimulation, in the expression of markers of the unfolded protein response (UPR, an ER stress reaction), nor in the levels of autophagy markers in response to rapamycin and bafilomycin A1 treatment. Finally, all 4 INPP5K mutant proteins tested, including p.lle363Thr in the SKICH domain, bound to GRP78 in a similar way to control INPP5K (Wiessner et al., 2017). In connection with the congenital muscular dystrophy observed in human INPP5K mutants, it is important to note that 3 single nucleotide polymorphisms (SNPs) located in intron 1 and 6 as well as in exon 12 of the porcine INPP5K gene have been associated with some meat traits, including muscle fiber number (Xiong et al., 2011, 2012). Furthermore, INPP5K gene-modified $\mathrm{Pps}^{\mathrm{Brdm} 1 /+}$ heterozygous mice had higher amounts of quadriceps muscle compared with wild-type mice (ljuin et al., 2008).

\section{INPP5K functions in cellular and animal models:}

The zebrafish as a model for the human congenital muscular dystrophy caused by biallelic mutations in the INPP5K gene:

Inpp5k is present in two copies in the zebrafish genome: inpp5ka and inpp5kb, with 48 and $44 \%$ identity to human INPP5K, respectively. At 1 and 2 days post-fertilization (dpf), mRNA quantification indicated that inpp5ka is respectively 7- and 16-fold higher expressed than inpp5kb (Osborn et al., 2017). In vivo experiments were conducted either by injecting inpp5ka-, inpp5kb- or inpp5ka- and Inpp5kb-specific morpholinos (MOs) in embryos (Osborn et al., 2017; Wiessner et al., 2017), or on a zebrafish line 
carrying an inpp5ka nonsense allele (p.GIn440*, resulting in a C-terminal 12 amino acids deletion, a probable hypomorph allele) (Yousaf et al., 2018). Injections of inpp5ka or inpp5ka and inpp5kb MOs in fertilized oocytes led to a phenotype that closely resembles the human presentation, including myopathy, reduced mobility, lens disorganization, microphtalmia, reduced growth, and microcephaly. In contrast, inpp5kb MOs alone showed only a very mild phenotype. Histologically, sparser and disorganized myofibers, altered sarcomere assembly, and reduced synaptic formation were observed in skeletal muscle of inpp5k knockdown embryos. In inpp5k morphans, the lens cortex was disorganized and cell nuclei were present in the center of the lens nucleus, a phenotype reminiscent of congenital cataracts in man. The p.GIn440* zebrafish line displayed a very similar lens phenotype, while the skeletal muscle phenotype was much less severe than observed in MOs-injected embryos (Yousaf et al., 2018).

Insulin signaling and insulin resistance:

As already mentioned above, according to ljuin and collaborators, INPP5K is localized at the cytosolic side of the ER membrane in non-stimulated conditions, directly interacting with ER luminal GRP78 (ljuin and Takenawa, 2012b, 2015a, 2015b, 2015c and 2016). Following insulin stimulation, the INPP5K-GRP78 complex migrates to the plasma membrane, where activated PAK1 competitively binds to INPP5K in place of GRP78, linking INPP5K to the complex of proteins associated with the insulin receptor. Binding of INPP5K to this complex through activated PAK1 leads to Ptdlns(3,4,5)P3 dephosphorylation, decreased AKT2, p70 S6 kinase and Glycogen Synthase activation, GLUT4 translocation, membrane ruffle formation and results in insulin signaling inactivation. Inversely, decreasing INPP5K expression in cells or in INPP5K gene-modified Pps ${ }^{\mathrm{Brdm} 1 /+}$ heterozygous mice resulted in an 
increased insulin signaling and sensitivity in muscle cells and, in $\mathrm{Pps}^{\mathrm{Brdm} 1 /+}$ mice, a reduced diet-induced obesity (ljuin and Takenawa, 2003, 2008; Xiong et al., 2009). Finally, INPP5K expression was increased in skeletal muscles isolated from high fat diet-fed mice and diabetic/obese $\mathrm{db} / \mathrm{db}$ mice, as compared to wild-type mice, linking INPP5K to these diseases (ljuin et al., 2015a). In this context, it is noteworthy that culture medium supplementation with a synthetic peptide containing the amino acids sequence of the PAK1 kinase domain which interacts with INPP5K increased insulin signaling in muscle cell lines, probably by interfering with the formation of the INPP5K-PAK1 complex (ljuin and Takenawa, 2015b).

ER stress, the unfolded protein response (UPR) and insulin resistance:

The UPR is triggered essentially in response to ER defects in N-linked glycosylation and disulfide bond formation, leading to accumulation of unfolded/misfolded proteins in the ER lumen (i.e. ER stress). ER stress can be secondary to intracellular alterations (e.g., calcium or redox imbalances, glucose deprivation), microenvironmental conditions (e.g., hypoglycemia, hypoxia, acidosis, viral infection), high-fat diet/obesity, a variety of natural compounds (e.g., thapsigargin, tunicamycin), and several prescription drugs. Physiological processes like a vigorous protein production and secretion (e.g. immunoglobulins in plasma cells, digestive enzymes in exocrine pancreas and intestine) or muscle cell contraction during intense/prolonged exercise also trigger the UPR. The GRP78 molecular chaperone, one of the INPP5K interacting proteins (see above), plays a central role in the UPR by binding to and inactivating 3 stress sensors present in the ER membrane: IRE1a, ATF6 and PERK. Briefly, accumulation of unfolded/misfolded proteins in the ER lumen displaces the GRP78 chaperone from these sensors to exposed hydrophobic residues on abnormally folded proteins. Activation of these 3 sensors results in fine in a 
decreased protein translation, increased production of molecular chaperones involved in protein folding, degradation of unfolded proteins by the proteasome and cell cycle arrest. If the UPR is prolonged or failed, cellular apoptosis occurs. ljuin and colleagues showed that UPR activation lead to an increased expression of INPP5K mRNA and protein in $\mathrm{C} 2 \mathrm{C} 12$ muscle cells and this contributed, as explained above, to decreased insulin signaling, i.e. insulin resistance. (ljuin et al., 2016). XBP1 transcription factor was involved in this increased expression. Increased skeletal muscle INPP5K expression was also observed in diet-induced and genetic models of obesity in mice where the UPR is chronically activated, suggesting a link between high-fat diet, obesity, the UPR (including XBP1), INPP5K expression and insulin resistance.

\section{ER structural integrity:}

Dong and collaborators elegantly demonstrated that INPP5K interacts with the Nterminus of ARL6IP1 at the cytosolic side of the ER membrane (Dong et al., 2018). Both proteins are enriched in highly motile, elongating peripheral ER tubules, relative to perinuclear ER sheets, and were absent from the nuclear membrane. Interestingly, knock-down of INPP5K or ARL6IP1 resulted in a similar decrease in ER tubules and expansion of ER sheets at the cell periphery. Both the INPP5K SKICH domain and 5phosphatase activity were shown to be essential to keep a normal ER structure. Yet, using GFP-PH PLCס1 as a fluorescent probe to monitor Ptdlns(4,5)P2 levels, no change in the localization and/or level of the fluorescent signal was detected in INPP5K knock-down cells (Dong et al., 2018). Defects in ER architecture and lower abundance of peripheral ER tubules were also observed in $C$. elegans neurons and human glioblastoma cell lines when INPP5K (or CIL-1, its $C$. elegans orthologue) was silenced (Dong et al., 2018; Ramos et al., 2020). Importantly, three CIL-1 mutations 
(I39T, Y261C, and I327T) corresponding to INPP5K mutations found in human muscular dystrophic patients did not rescue the ER morphology phenotype when expressed in C. elegans CIL-1-deficient neurons, on the contrary to the wild type CIL1 protein (Dong et al., 2018).

Myoblasts differentiation and control of the IGF-II autocrine regulatory loop:

Expression of INPP5K mRNA and protein increased during C2C12 cell differentiation into myotubes. In addition, MyoD, a transcription factor essential for myoblast specification during embryogenesis and in adults, induced INPP5K mRNA expression in these cells, partly via cis-acting elements in the INPP5K promoter (Xiong et al., 2011; ljuin and Takenawa, 2012a). C2C12 cell differentiation into myotubes was decreased when INPP5K was overexpressed and, inversely, endogenous INPP5K silencing was associated with increased myotubes formation. A model was proposed by ljuin and colleagues where INPP5K controls the IGF-II autocrine regulatory loop and thus myoblast differentiation (ljuin and Takenawa, 2012a). Indeed, during myoblast differentiation, IGF-II up-regulates its own gene expression through the PI3kinase/Ptdlns(3,4,5)P3/AKT/mTOR signaling pathway, leading to IGF-II production and secretion in the extracellular space. Secreted IGF-II binds to IGF-1 receptor on myoblasts and initiate an autocrine/paracrine loop that plays a major role during differentiation (Ren et al., 2008; Duan et al., 2010; Jiao et al., 2013, and references therein). In the proposed model, INPP5K dephosphorylates Ptdlns(3,4,5)P3 in the IGF-II signaling pathway, leading to decreased AKT/mTOR phosphorylation and IGF-II mRNA and protein production, acting thus as a brake on this positive autocrine loop. This mechanism could explain why INPP5K overexpression in $\mathrm{C} 2 \mathrm{C} 12$ cells decreased myoblast differentiation and why decreased endogenous INPP5K expression in these cells and in INPP5K gene- 
modified $P p s^{B r d m 1 /+}$ heterozygous mice led to significantly increased differentiation into myotubes and quadriceps muscle weight compared to controls (ljuin et al., 2008, 2012a).

Cytoskeleton organization, cell adhesion and migration:

Following cell stimulation by growth factors like Epidermal Growth factor (EGF) or integrin activation during cell adhesion, INPP5K translocates from the ER to the plasma membrane and co-localized with submembranous actin and membrane ruffles (Gurung et al., 2003; ljuin and Takenawa, 2003; Davies et al., 2015; Ramos et al., 2019b). In these conditions, decreasing INPP5K expression in cancer cell lines had no impact on cell proliferation or survival, but resulted in increased Ptdlns(4,5)P2 levels and decreased cell migration (Davies et al., 2015; Ramos et al., 2019b). By contrast, INPP5K overexpression suppressed growth factor-induced Ptdlns(3,4,5)P3/AKT signaling and cell growth, and reduced the recruitment of $\mathrm{PI}(4,5) \mathrm{P} 2$-binding proteins to the plasma membrane, lamellipodia formation and Talin incorporation into focal adhesions (Davies et al., 2015).

Renal osmoregulation:

Transgenic mice ubiquitously overexpressing tagged mouse INPP5K protein presented defects in water metabolism characterized by a reduced plasma osmolality at baseline, a delayed urinary water excretion following a water load, and an increased acute response to vasopressin. These defects were associated with the expression of the Inpp5k transgene in renal collecting ducts and with alterations in the arginine vasopressin/aquaporin-2 signalling pathway in this tubular segment. Analysis in a mouse collecting duct mCCD cell line revealed that INPP5K overexpression lead to increased expression of the arginine vasopressin receptor type 2 and increased cAMP response to arginine vasopressin, providing a basis for 
increased aquaporin-2 expression and plasma membrane localization with increased osmotically-induced water transport. Altogether, these results indicated that INPP5K is important for the control of the arginine vasopressin/aquaporin-2 signalling pathway and water transport in kidney collecting ducts.

INPP5K association with other biological processes and human diseases: As mentioned above, INPP5K mRNA expression was found either increased or decreased in cancer, depending on the cancer cell type, but the exact consequence on cancer physiopathology remains unclear. By contrast, Ramos and colleagues recently showed that INPP5K depletion in the U-87 MG glioblastoma cell line resulted in increased viability and decreased apoptosis, and that these cells generated significantly larger tumors when injected in SCID mice, compared with controls (Ramos et al., 2020).

Association studies also revealed links between the INPP5K gene and autophagy (Zirin et al., 2015), Parkinson (Nazeri et al., 2015; Zhu et al., 2018; Liu et al., 2019) and Alzheimer diseases (Rahman et al., 2020).

\section{Conclusion, questions and future directions:}

In 2017, mutations in INPP5K have been detected in patients with a rare form of autosomal recessive congenital muscular dystrophy. All causative INPP5K alleles detected so far result in decreased catalytic activity or altered subcellular localization of the protein. Muscle pathology is largely non-specific in patients as in inpp5k knockdown zebrafish, a model used to mimic the human disease. Unfortunately, the pathogenic mechanism of this muscle disease remains totally unknown. In particular, no difference in AKT phosphorylation was detected between patients and control fibroblasts in response to IGF-II stimulation, even though numerous cellular studies seemed to point this growth factor (or insulin)/PI3kinase/ Ptdlns(3,4,5)P3/AKT axis as 
a good candidate for the pathogenic mechanism of this disease. The precise intracellular localization of INPP5K in muscle cells will probably give some ideas about the mechanism of action of INPP5K in this cell type and the physiopathology of this muscular dystrophy. Precisions about the human phenotype are also needed: why do patients with the same INPP5K mutation have so variable penetrance of muscular dystrophy and intellectual disability? Are INPP5K patients also insulin hypersensitive, like INPP5K gene-modified $P p s^{B r d m 1 /+}$ heterozygous mice? Conversely, do these INPP5K heterozygous mice have muscle histological alterations similar to INPP5K patients or INPP5K-depleted zebrafish? More generally, in light of the information in this review, it will be important to discover the specific physiological and functional relevance, if any, of human 42.7 and $36 \mathrm{kDa}$ as well as mouse 51,42 and $36 \mathrm{kDa}$ INPP5K proteins, to further analyze the interaction of INPP5K with MAD2L1BP and the transfer of this complex into the nucleus, as well as the physiological and functional role of INPP5K in this specific subcellular compartment, in relation with nuclear Ptdlns(4,5)P2 levels. In the same line of thought, the impact of INPP5K inactivation on ER functional integrity, beside structural integrity (e.g. protein and lipid syntheses, protein glycosylation, calcium signaling,...) and on ER phosphoinositide levels should also be investigated. Finally, ljuin and colleagues reported that INPP5K gene-modified $P p s^{\mathrm{Brdm} 1 / 1 / \mathrm{rdm} 1}$ homozygous mice died early during embryonic life of unknown causes (ljuin et al., 2008). We have generated Inpp5k floxed and then Inpp5 $k^{\Delta / \Delta}$ mice and we could confirm that the latter mice died during embryonic life, as $P p s^{\text {Brdm1/Brdm1 }}$ mice. Our floxed mice as well as those recently generated by McCabe (McCabe et al., 2019) will certainly help to answer to most of the above questions and will probably allow discovering the exact INPP5K function during eye development and in lens cortex, neurons and brain as 
well as skeletal muscle. The analysis of INPP5K floxed mice will probably reveal a wider phenotype than in INPP5K patients, with alterations involving more biological processes and organs, given that human INPP5K mutations do not completely abolish the 5-phosphatase catalytic activity or function.

Altogether, the analysis of this original phosphoinositide 5-phosphatase will certainly give some surprising and interesting results about its role and its Ptdlns $(4,5) \mathrm{P} 2$ and Ptdlns $(3,4,5) \mathrm{P} 3$ substrates in physiology and physiopathology in many organs, cells and subcellular localizations. 


\section{Disclosure of conflicts of interest:}

The authors disclose no potential conflicts of interest. All authors approved the final manuscript.

\section{Acknowledgments:}

C-AVC and BM are supported by a FRIA grant from the belgian FNRS. CD is a research associate of the belgian FNRS. 


\section{References :}

Blackstone, C., O'Kane, C. J., Reid, E., 2011. Hereditary spastic paraplegias: membrane traffic and the motor pathway. Nat. Rev. Neurosci.12: 31-42.

Blero, D., Payrastre, B., Schurmans, S., Erneux, C.,2007. Phosphoinositide phosphatases in a network of signaling reactions. Pflug Arch. Eur. J. Phy. 455: 31-44.

Davies, E. M., Kong, A. M., Tan, A., Gurung, R., Sriratana, A., Bukczynska, P. E., Ooms, L. M., McLean, C. A., Tiganis, T., Mitchell, C. A., 2015. Differential SKIP expression in PTEN-deficient glioblastoma regulates cellular proliferation and migration. Oncogene 34: 3711-27.

Dong, R., Zhu T., Benedetti L., Gowrishankar S., Deng H., Cai Y., Wang X., Shen K., De Camilli P., 2018. The inositol 5-phosphatase INPP5K participates in the fine control of ER organization. J. Cell. Biol. 217: 3577-3592.

Duan, C., Ren, H., Gao, S., 2010. Insulin-like growth factors (IGFs), IGF receptors, and IGF-binding proteins: Roles in skeletal muscle growth and differentiation. Gen. Comp. Endocrinol. 167: 344-51.

Falkenburger, B. H., Jensen, J. B., Dickson, E. J., Suh, B.-C., Hille, B., 2010.

Phosphoinositides: lipid regulators of membrane proteins. J. Physiol. 588.17: 31793185. 
Flaig, T. W., Salzmann-Sullivan, M., Su, L. J., Zhang, Z., Joshi, M., Gijon, M. A., Kim, J., Arcaroli, J. J., Van Bokhoven, A., Lucia, M. S., La Rosa, F. G., Schlaepfer, I. R., 2017. Lipid catabolism inhibition sensitizes prostate cancer cells to antiandrogen blockade. Oncotarget 8: 56051-56065.

Gao, L., Ye, M., Lu, X., Huang, D., 2017. Hybrid method based on information gain and support vector machine for gene selection in cancer classification. Genomics Proteomics Bioinform. 15: 389-395.

Grabon, A., Bankaitis, V. A., McDermott, M. I., 2019. The interface between phosphatidylinositol transfer protein function and phosphoinositide signaling in higher eukaryotes. J. Lipid Res. 60: 242-268.

Gurung, R., Tan, A., Ooms, L. M., McGrath, M. J., Huysmans, R. D., Munday, A. D., Prescott, M., Whisstock, J. C., Mitchell, C. A., 2003. Identification of a novel domain in two mammalian inositol-polyphosphate 5-phosphatases that mediates membrane ruffle localization. The inositol 5-phosphatase skip localizes to the endoplasmic reticulum and translocates to membrane ruffles following epidermal growth factor stimulation. J. Biol. Chem. 278: 11376-85.

Hedberg Oldfors, C., Dios, D. G., Linder, A., Visuttijai, K., Samuelson, E., Karlsson, S., Nilsson, S., Behboudi, A., 2015. Analysis of an independent tumor suppressor locus telomeric to Tp53 suggested Inpp5k and Myo1c as novel tumor suppressor gene candidates in this region. BMC Genet. 16:80. doi: 10.1186/s12863-015-0238-4. 
Hung, C. S., Lin, Y. L., Wu, C. I., Huang, C. J., Ting, L. P., 2009. Suppression of hepatitis B viral gene expression by phosphoinositide 5-phosphatase SKIP. Cell. Microbiol. 11: 37-50.

ljuin, T., Mochizuki, Y., Fukami, K., Funaki, M., Asano, T., Takenawa, T., 2000. Identification and characterization of a novel inositol polyphosphate 5-phosphatase. J. Biol. Chem. 275: 10870-5.

ljuin, T., Takenawa, T., 2003. SKIP negatively regulates insulin-induced GLUT4 translocation and membrane ruffle formation. Mol. Cell. Biol. 23: 1209-20.

Ijuin, T., Yu, Y. E., Mizutani, K., Pao, A., Tateya, S., Tamori, Y., Bradley, A., Takenawa, T., 2008. Increased insulin action in SKIP heterozygous knockout mice. Mol. Cell. Biol. 28: 5184-95.

Ijuin, T., Takenawa, T., 2012a. Role of phosphatidylinositol 3,4,5-trisphosphate (PIP3) 5-phosphatase skeletal muscle- and kidney-enriched inositol polyphosphate phosphatase (SKIP) in myoblast differentiation. J. Biol. Chem. 287: 31330-41.

ljuin, T., Takenawa, T., 2012b. Regulation of insulin signaling by the phosphatidylinositol 3,4,5-triphosphate phosphatase SKIP through the scaffolding function of Pak1. Mol. Cell. Biol. 32: 3570-84. 
Ijuin, T., Hosooka, T., Takenawa, T., 2015a. Phosphatidylinositol 3,4,5-Trisphosphate Phosphatase SKIP Links Endoplasmic Reticulum Stress in Skeletal Muscle to Insulin Resistance. Mol. Cell. Biol. 36: 108-18.

Ijuin, T., Takenawa, T., 2015b. Improvement of insulin signaling in myoblast cells by an addition of SKIP-binding peptide within Pak1 kinase domain. Biochem. Biophys. Res. Commun. 456: 41-6.

Ijuin, T., Hatano, N., Hosooka, T., Takenawa, T., 2015c. Regulation of insulin signaling in skeletal muscle by PIP3 phosphatase, SKIP, and endoplasmic reticulum molecular chaperone glucose-regulated protein 78. Biochim. Biophys. Acta. 1853: 3192-201.

Ijuin, T., Hatano, N., Takenawa, T., 2016. Glucose-regulated protein 78 (GRP78) binds directly to PIP3 phosphatase SKIP and determines its localization. Genes Cells 21: 457-65.

Jiao, S., Ren, H., Li, Y., Zhou, J., Duan, C., Lu, L., 2013. Differential regulation of IGF-I and IGF-II gene expression in skeletal muscle cells. Mol. Cell. Biochem. 373: 107-113.

Jones, J., Otu, H., Spentzos, D., Kolia, S., Inan, M., Beecken, W. D., Fellbaum, C., Gu, X., Joseph, M., Pantuck, A. J., Jonas, D., Libermann, T. A., 2005. Gene signatures of progression and metastasis in renal cell cancer. Clin. Cancer Res., 11: 5730-5739. 
Liu, G., Zhao, Y., Sun, J. Y., Sun, B. L., 2019. Parkinson's Disease Risk Variant rs1109303 Regulates the Expression of INPP5K and CRK in Human Brain. Neurosci. Bull. 35: 365-368.

McCabe, C. V., Codner, G. F., Allan, A. J., Caulder, A., Christou, S., Loeffler, J., Mackenzie, M., Malzer, E., Mianné, J., Pike, F. J., Hutchison, M., Stewart, M. E., Gates, H., Wells, S., Sanderson, N. D., Teboul, L., 2019. Application of long-read sequencing for robust identification of correct alleles in genome edited animals. BioRxiv doi: https://doi.org/10.1101/838193.

Nazeri, A., Roostaei, T., Sadaghiani, S., Chakravarty, M. M., Eberly, S., Lang, A. E., Voineskos, A. N., 2015. Genome-wide variant by serum urate interaction in Parkinson's disease. Ann. Neurol. 78: 731-41.

Nizon, M., Küry, S., Péréon, Y., Besnard, T., Quinquis, D., Boisseau, P., Marsaud, T., Magot, A., Mussini, J. M., Mayrargue, E., Barbarot, S., Bézieau, S., Isidor, B., 2018. ARL6IP1 mutation causes congenital insensitivity to pain, acromutilation and spastic paraplegia. Clin. Genet. 93: 169-172.

Novarino, G., Fenstermaker, A. G., Zaki, M. S., Hofree, M., Silhavy, J. L., Heiberg, A. D., Abdellateef, M., Rosti, B., Scott, E., Mansour, L., Masri, A., Kayserili, H., Al-Aama, J. Y., Abdel-Salam, G. M. H., Karminejad, A., Kara, M., Kara, B., Bozorgmehri, B., Ben-Omran, T., Mojahedi, F., El Din Mahmoud, I. G., Bouslam, N., Bouhouche, A., Benomar, A., Hanein, S., Raymond, L., Forlani, S., Mascaro, M., Selim, L., Shehata, 
N., Al-Allawi, N., Bindu, P. S., Azam, M., Gunel, M., Caglayan, A., Bilguvar, K., Tolun, A., Issa, M. Y., Schroth, J., Spencer, E. G., Rosti, R. O., Akizu, N., Vaux, K. K., Johansen, A., Koh, A. A., Megahed, H., Durr, A., Brice, A., Stevanin, G., Gabriel, S. B., Ideker, T., Gleeson, J. G., 2014. Exome sequencing links corticospinal motor neuron disease to common neurodegenerative disorders. Science. 343: 506-511.

Osborn, D. P. S., Pond, H. L., Mazaheri, N., Dejardin, J., Munn, C. J., Mushref, K., Cauley, E. S., Moroni, I., Pasanisi, M. B., Sellars, E. A., Hill, R. S., Partlow, J. N., Willaert, R. K., Bharj, J., Malamiri, R. A., Galehdari, H., Shariati, G., Maroofian, R., Mora, M., Swan, L. E., Voit, T., Conti, F. J., Jamshidi, Y., Manzini, M. C., 2017. Mutations in INPP5K Cause a Form of Congenital Muscular Dystrophy Overlapping Marinesco-Sjögren Syndrome and Dystroglycanopathy. Am. J. Hum. Genet. 100: 537-545.

Pernot, E., Terryn, S., Cheong, S. C., Markadieu, N., Janas, S., Blockmans, M., Jacoby, M., Pouillon, V., Gayral, S., Rossier, B. C., Beauwens, R., Erneux, C., Devuyst, O., Schurmans, S., 2011. The inositol Inpp5k 5-phosphatase affects osmoregulation through the vasopressin-aquaporin 2 pathway in the collecting system. Pflugers Arch. 462: 871-83.

Rahman, P., Huysmans, R. D., Wiradjaja, F., Gurung, R., Ooms, L. M., Sheffield, D. A., Dyson, J. M., Layton, M. J., Sriratana, A., Takada, H., Tiganis, T., Mitchell, C. A., 2011. Silencer of death domains (SODD) inhibits skeletal muscle and kidney enriched inositol 5-phosphatase (SKIP) and regulates phosphoinositide 3-kinase (PI3K)/Akt signaling to the actin cytoskeleton. J. Biol. Chem. 286: 29758-70. 
Rahman, M. R., Islam, T., Zaman, T., Shahjaman, M., Karim, M. R., Huq, F., Quinn, J. M. W., Holsinger, R. M. D., Gov, E., Moni, M. A., 2020. Identification of biomarkers and pathways to identify novel therapeutic targets in Alzheimer's disease: Insights from a systems biomedicine perspective. Genomics 112: 1290-1299.

Ramos, A. R., Elong Edimo, W., Erneux, C., 2018. Phosphoinositide 5-phosphatase activities control cell motility in glioblastoma: Two phosphoinositides $\mathrm{PI}(4,5) \mathrm{P} 2$ and PI(3,4)P2 are involved. Adv. Biol. Regul. 67:40-48. doi: 10.1016/j.jbior.2017.09.001.

Ramos, A. R., Ghosh, S., Erneux, C., 2019a. The impact of phosphoinositide 5phosphatases on phosphoinositides in cell function and human disease. J. Lipid Res. 60: 276-286.

Ramos, A. R., Ghosh, S., Dedobbeleer, M., Robe, P. A., Rogister, B., Erneux, C., 2019b. Lipid phosphatases SKIP and SHIP2 regulate fibronectin-dependent cell migration in glioblastoma. FEBS J. 286: 1120-1135.

Ramos, A. R., Ghosh, S., Suhel, T., Chevalier, C., Obeng, E. O., Fafilek, B., Krejci, P., Beck, B., Erneux, C., 2020. Phosphoinositide 5-phosphatases SKIP and SHIP2 in ruffles, the endoplasmic reticulum and the nucleus: An update. Adv. Biol. Regul. 75:100660. doi: 10.1016/j.jbior.2019.100660. 
Ren, H., Yin, P., Duan, C.J., 2008. IGFBP-5 regulates muscle cell differentiation by binding to IGF-II and switching on the IGF-II auto-regulation loop. Cell Biol. 182: 97991.

Rual, J.F., Venkatesan, K., Hao, T., Hirozane-Kishikawa, T., Dricot, A., Li, N., Berriz, G. F., Gibbons, F. D., Dreze, M., Ayivi-Guedehoussou, N., Klitgord, N., Simon, C., Boxem, M., Milstein, S., Rosenberg, J., Goldberg, D. S., Zhang, L. V., Wong, S. L., Franklin, G., Li, S., Albala, J. S., Lim, J., Fraughton, C., Llamosas, E., Cevik, S., Bex, C., Lamesch, P., Sikorski, R. S., Vandenhaute, J., Zoghbi, H. Y., Smolyar, A., Bosak, S., Sequerra, R., Doucette-Stamm, L., Cusick, M. E., Hill, D. E., Roth, F. P., Vidal, M., 2005. Towards a proteome-scale map of the human protein-protein interaction network. Nature 437: 1173-8.

Schmid, A. C., Wise, H. M., Mitchell, C. A., Nussbaum, R., Woscholski, R., 2004. Type II phosphoinositide 5-phosphatases have unique sensitivities towards fatty acid composition and head group phosphorylation. FEBS Lett. 576: 9-13.

Wiessner, M., Roos, A., Munn, C. J., Viswanathan, R., Whyte, T., Cox, D., Schoser, B., Sewry, C., Roper, H., Phadke, R., Marini Bettolo, C., Barresi, R., Charlton, R., Bönnemann, C. G., Abath Neto, O., Reed, U. C., Zanoteli, E., Araújo Martins Moreno, C., Ertl-Wagner, B., Stucka, R., De Goede, C., Borges da Silva, T., Hathazi, D., Dell'Aica, M.., Zahedi,R. P., Thiele, S., Müller, J., Kingston, H., Müller, S., Curtis, E., Walter, M. C., Strom, T. M., Straub, V., Bushby, K., Muntoni, F., Swan, L. E., Lochmüller, H., Senderek, J., 2017. Mutations in INPP5K, Encoding a 
Phosphoinositide 5-Phosphatase, Cause Congenital Muscular Dystrophy with Cataracts and Mild Cognitive Impairment. Am. J. Hum. Genet. 100: 523-536.

Wiradjaja, F., Ooms, L. M., Whisstock, J. C., McColl, B., Helfenbaum, L., Sambrook, J. F., Gething, M. J., Mitchell, C. A., 2001. The yeast inositol polyphosphate 5phosphatase Inp54p localizes to the endoplasmic reticulum via a C-terminal hydrophobic anchoring tail: regulation of secretion from the endoplasmic reticulum. J. Biol. Chem. 276: 7643-53.

Xiong, Q., Deng, C. Y., Chai, J., Jiang, S. W., Xiong, Y. Z., Li, F. E., Zheng, R., 2009. Knockdown of endogenous SKIP gene enhanced insulin-induced glycogen synthesis signaling in differentiating C2C12 myoblasts. BMB Rep. 42: 119-24.

Xiong, Q., Chai, J., Zhang, P. P., Wu, J., Jiang, S. W., Zheng, R., Deng, C. Y., 2011. MyoD control of SKIP expression during pig skeletal muscle development. Mol. Biol. Rep. 38: 267-74.

Xiong, Q., Chai, J., Deng, C., Jiang, S., Liu, Y., Huang, T., Suo, X., Zhang, N., Li, X., Yang, Q., Chen, M., Zheng, R., 2012. Characterization of porcine SKIP gene in skeletal muscle development: polymorphisms, association analysis, expression and regulation of cell growth in C2C12 cells. Meat Sci. 92: 490-7.

Yamamoto, Y., Yoshida, A., Miyazaki, N., Iwasaki, K., and Sakisaka, T., 2014. Arl6IP1 has the ability to shape the mammalian ER membrane in a reticulon-like fashion. Biochem. J. 458: 69-79. 
Yang, Y., Wang, G., Huang, X., Du, Z., 2015. Crystallo-graphic and modelling studies suggest that the $\mathrm{SKICH}$ domains from different protein families share a common Iglike fold but harbour substantial structural variations. J. Bio-mol. Struct. Dyn. 33: $1385-1398$.

Yousaf, S., Sheikh, S. A., Riazuddin, S., Waryah, A. M., Ahmed, Z. M., 2018. INPP5K variant causes autosomal recessive congenital cataract in a Pakistani family. Clin. Genet. 93: 682-686.

Zhu, W., Luo, X., Adnan, A., Yu, P., Zhang, S., Huo, Z., Xu, Q., Pang, H., 2018. Association analysis of NUCKS1 and INPP5K polymorphism with Parkinson's disease. Genes Genet. Syst. 93: 59-64.

Zirin, J., Nieuwenhuis, J., Samsonova, A., Tao, R., Perri-mon, N., 2015. Regulators of autophagosome formation in Drosophila muscles. PLoS Genet. 11, e1005006. 


\section{Figure Legend:}

Figure 1: The human INPP5K gene and protein, as well as mutations discovered in patients.

The top panel represents the relationship between exons (blue boxes) and the 5phosphatase and SKICH domains of the INPP5K protein. White boxes are 5' and 3' untranslated regions. The central panel represents the different domains of the Rasa3 protein with their known functions. The lower panel shows the 9 mutations discovered so far in patients with a rare form of autosomal recessive congenital muscular dystrophy. 
DNA :

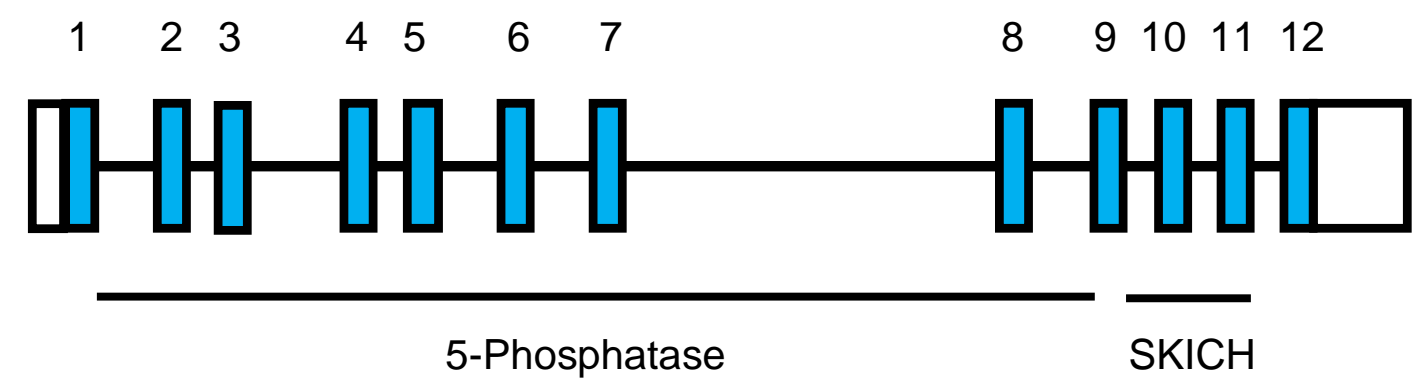

PROTEIN :

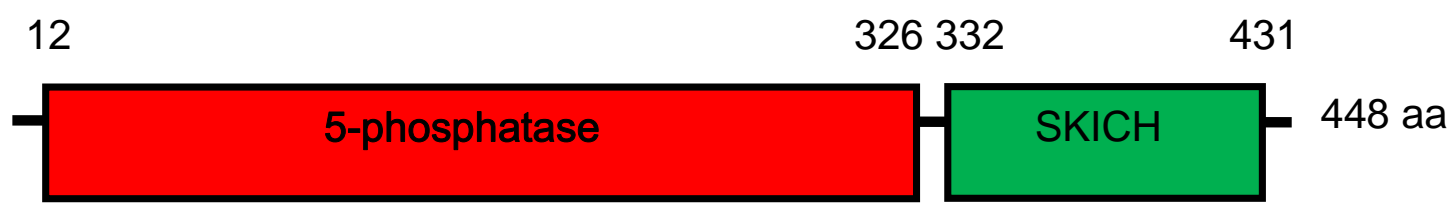

substrates: Ptdlns(4,5) $\mathrm{P}_{2}$ and $\mathrm{Ptdlns}(3,4,5) \mathrm{P}_{3}$ protein-protein interaction subcellular localization

\section{MUTATIONS:}

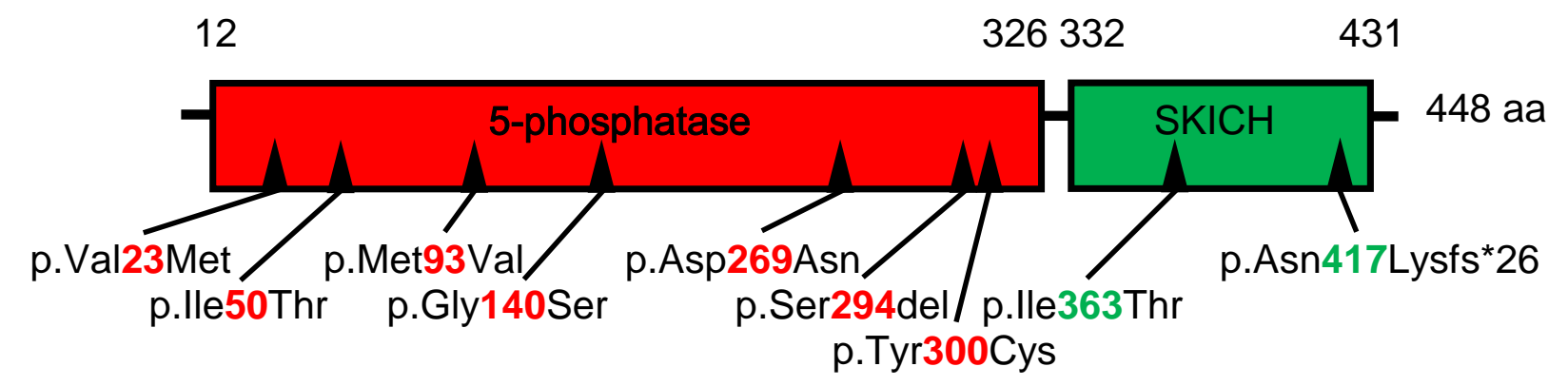

